\title{
Other Iatrogenic Immunodeficiency-associated Lymphoproliferative Disorder Presenting as Primary Bone Lymphoma in a Patient with Rheumatoid Arthritis
}

\author{
Kazuya Ishiguro ${ }^{1}$, Toshiaki Hayashi ${ }^{1}$, Yuka Aoki ${ }^{1}$, Rieko Murakami ${ }^{2}$, \\ Hiroshi Ikeda ${ }^{1}$ and Tadao Ishida ${ }^{1}$
}

\begin{abstract}
Primary bone lymphoma (PBL) is a rare disorder. We herein present a case of other iatrogenic immunodeficiency-associated lymphoproliferative disorder (OIIA-LPD) presenting as PBL. A 63-year-old woman was diagnosed with rheumatoid arthritis and had been treated with methotrexate for seven years. Two months before admission, she suffered from pain in the limbs. Magnetic resonance imaging revealed multiple irregular lesions in the bones of the limbs, which showed an uptake of ${ }^{18} \mathrm{~F}-\mathrm{FDG}$ on positron emission tomography. A biopsy of the right radius revealed diffuse large B-cell lymphoma, leading to the diagnosis of OIIA-LPD. She received rituximab-containing regimens resulting in a complete response.
\end{abstract}

Key words: primary bone lymphoma (PBL), other iatrogenic immunodeficiency-associated lymphoproliferative disorder (OIIA-LPD), methotrexate (MTX), positron emission tomography (PET), bone biopsy, rituximab

(Intern Med 55: 2259-2264, 2016)

(DOI: 10.2169/internalmedicine.55.6684)

\section{Introduction}

Primary bone lymphoma (PBL) is a rare disorder. It accounts for $5 \%$ of extranodal lymphomas (1-6), less than $1 \%$ of all non-Hodgkin's lymphomas $(3,6,7)$, and $3 \%$ of all primary malignant bone tumors $(2,5,8)$. Patients with rheumatoid arthritis (RA) tend to develop lymphoproliferative disorders (LPD) at a frequency of 2.0-5.5 times higher than that in the general population. However, its exact frequency in lymphomas is unknown $(9,10)$. When LPD is diagnosed in patients treated with immunosuppressive therapy, it is categorized as other iatrogenic immunodeficiency-associated lymphoproliferative disorder (OIIA-LPD) according to the World Health Organization (WHO) classification of tumors of the hematopoietic and lymphoid tissues. We herein present a case of RA with OIIA-LPD presenting as PBL. This case is helpful in the management of OIIA-LPD presenting as PBL due to its rarity and difficulty to diagnose and evaluate the response to therapy.

\section{Case Report}

A 63-year-old woman was referred to our hospital for the treatment of lymphoma. The patient was previously diagnosed with RA, which had been treated with methotrexate (MTX) for seven years. She had been asymptomatic until two months before this admission when pain in her limbs developed. Her rheumatologist suspected the exacerbation of RA; however, no physical or laboratory findings supported this suspicion. Magnetic resonance imaging (MRI) revealed multiple irregular lesions in the femurs, tibias, patellae, humeri, and radiuses (Fig. 1). These lesions were hypointense on the T1-weighted image and hyperintense on T2-weighted, fat suppression, and diffusion-weighted images. Positron emission tomography/computed tomography (PET/CT) re-

${ }^{1}$ Department of Gastroenterology, Rheumatology, and Clinical Immunology, Sapporo Medical University School of Medicine, Japan and ${ }^{2}$ Department of Rheumatology, JR Sapporo Hospital, Japan

Received for publication October 11, 2015; Accepted for publication December 2, 2015

Correspondence to Dr. Kazuya Ishiguro, k.ishiguro@sapmed.ac.jp 


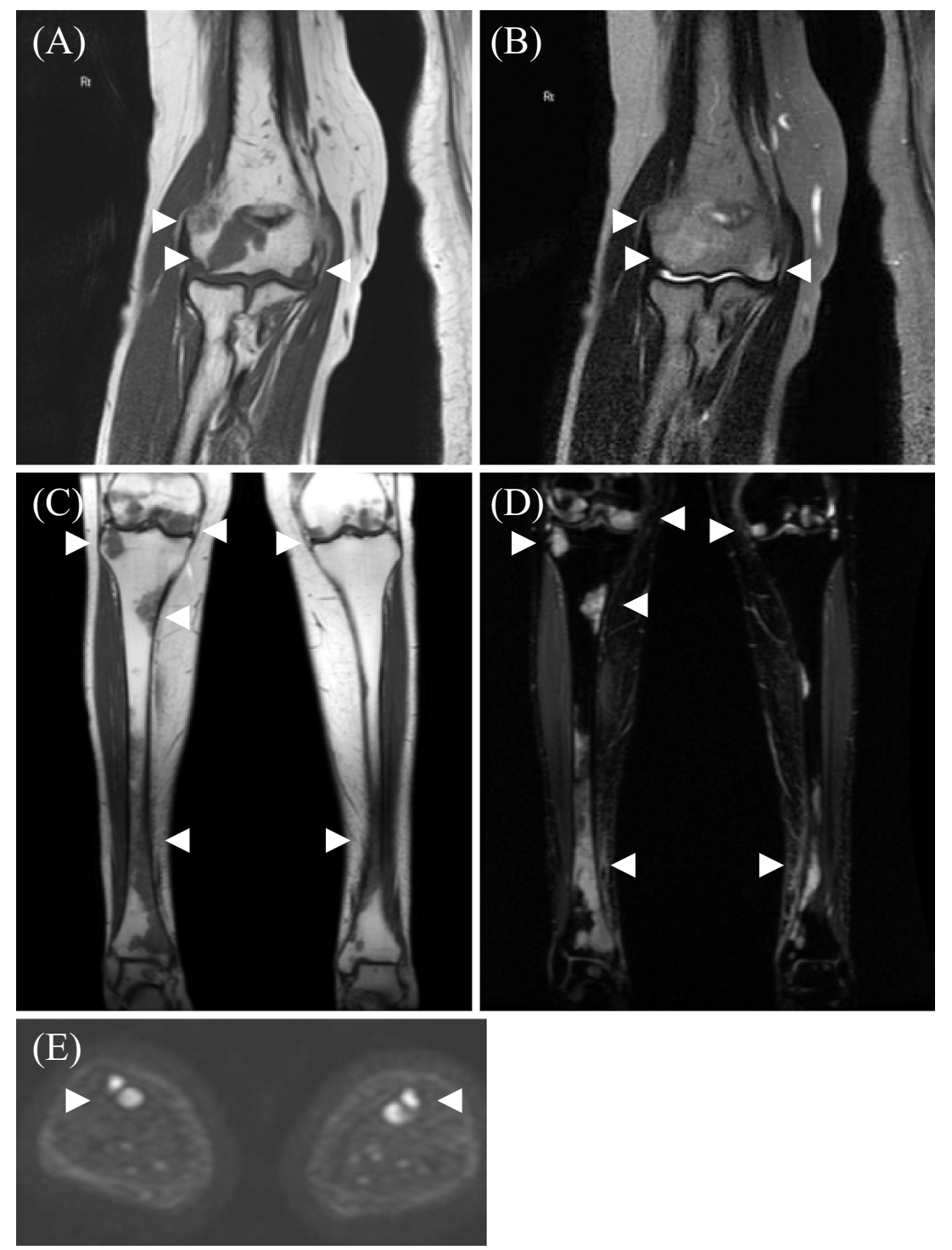

Figure 1. MRI of the right elbow revealed irregular hypointense lesions on the T1-weighted image (A) and irregular hyperintense lesions on the T2-weighted image (B). MRI of the femurs and tibias also revealed irregular hypointense lesions on the T1-weighted image $(C)$ and irregular hyperintense lesions on the fat suppression (D) and diffusion-weighted images (E). Each lesion is indicated by arrowheads.

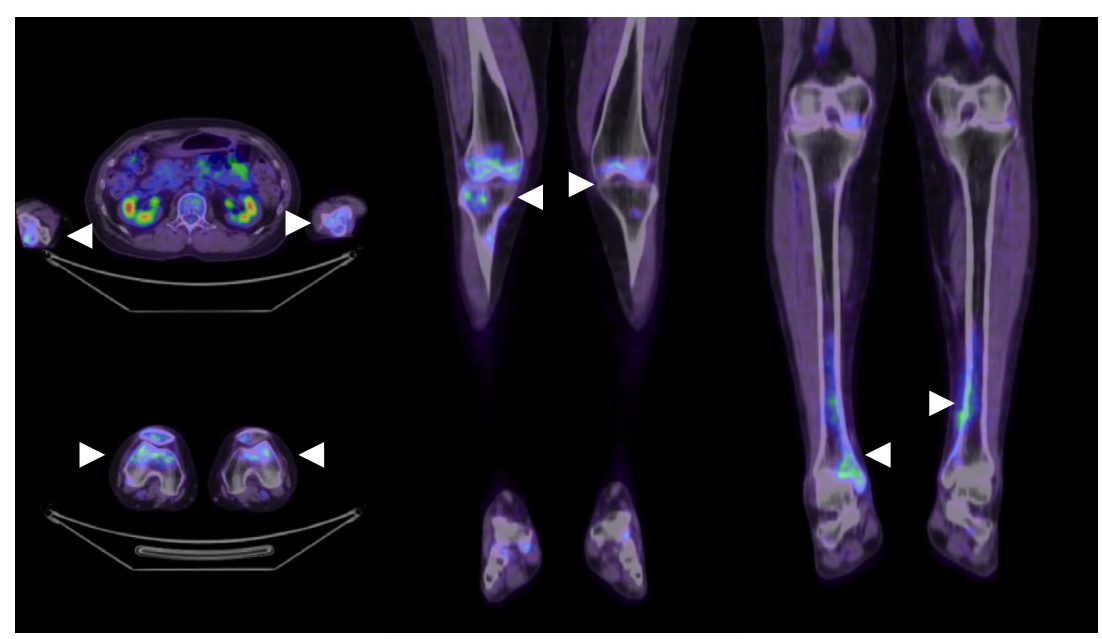

Figure 2. PET/CT revealed an uptake of ${ }^{18}$ F-FDG in the lesions. Each lesion is indicated by arrowheads. 

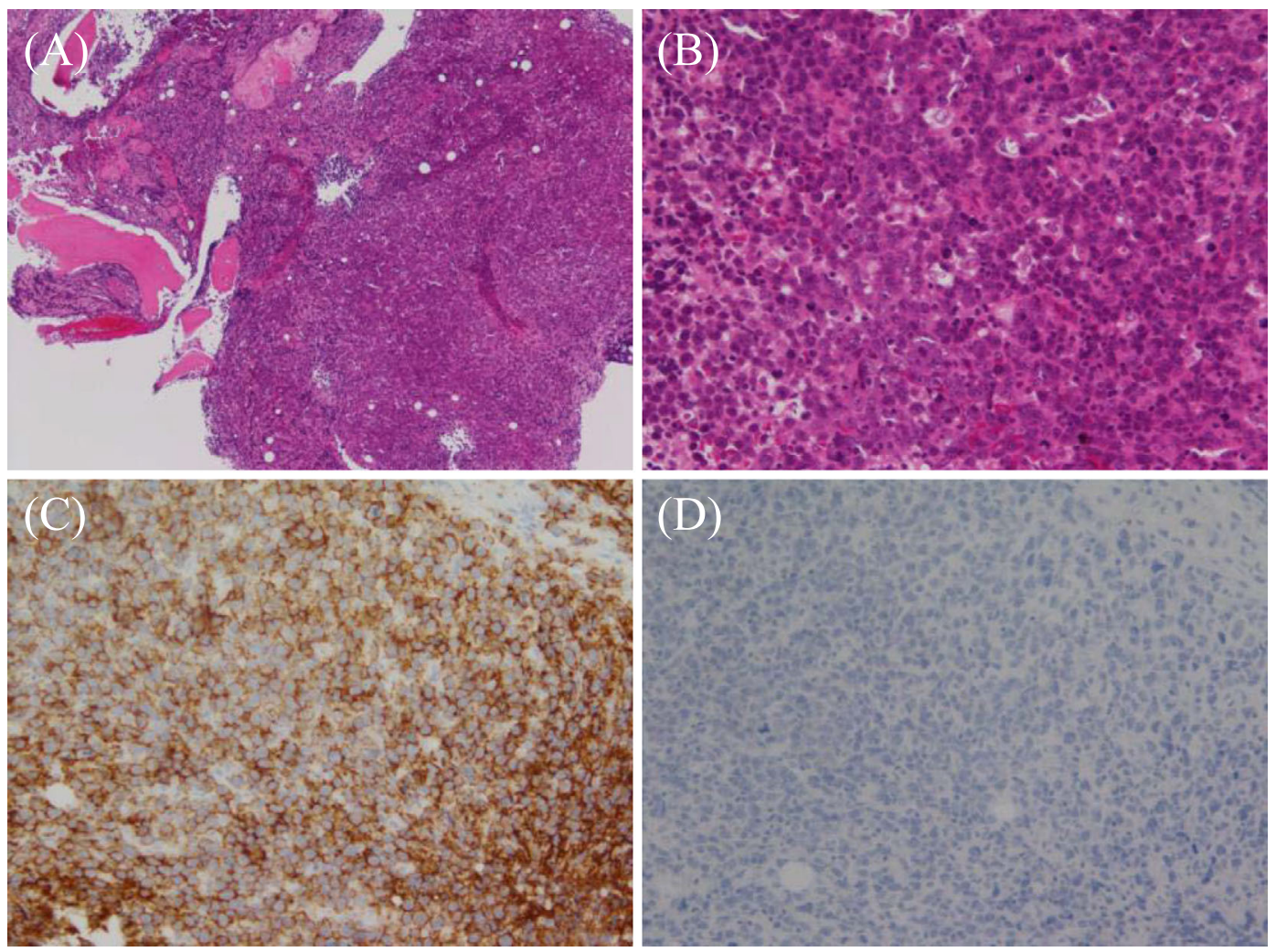

Figure 3. Large lymphoma cells with atypical large nuclei, including clear nucleoli and rough chromatin, showed diffuse proliferation surrounding the trabecula on a bone biopsy from the right radius. Lymphoma cells were CD20-positive and CD3-negative. (A) Hematoxylin and Eosin (H\&E) staining (40x), (B) H\&E staining (200x), (C) CD20 stain (200x), and (D) CD3 stain (200x).

Table. Laboratory Data on Admission.

\begin{tabular}{|c|c|c|c|}
\hline WBC & $8.7 \times 10^{3} \quad / \mathrm{mL}$ & $\mathrm{Na}$ & $141 \mathrm{mEq} / \mathrm{L}$ \\
\hline $\mathrm{RBC}$ & $410 \times 10^{4} \quad / \mathrm{mL}$ & $\mathrm{K}$ & $4.0 \mathrm{mEq} / \mathrm{L}$ \\
\hline $\mathrm{Hb}$ & $13.2 \mathrm{~g} / \mathrm{dL}$ & $\mathrm{Cl}$ & $105 \mathrm{mEq} / \mathrm{L}$ \\
\hline Plt & $19.4 \times 10^{4} \quad / \mathrm{mL}$ & $\mathrm{Ca}$ & $9.6 \mathrm{mg} / \mathrm{dL}$ \\
\hline \multirow[t]{2}{*}{ PT-INR } & 1.01 & CRP & $<0.10 \mathrm{mg} / \mathrm{dL}$ \\
\hline & & SIL-2R & $321 \mathrm{U} / \mathrm{mL}$ \\
\hline TP & $7.0 \mathrm{~g} / \mathrm{dL}$ & & \\
\hline Alb & $4.0 \mathrm{~g} / \mathrm{dL}$ & & \\
\hline T-Bil & $1.3 \mathrm{mg} / \mathrm{dL}$ & & \\
\hline CK & $38 \mathrm{IU} / \mathrm{L}$ & & \\
\hline AST & $20 \mathrm{IU} / \mathrm{L}$ & & \\
\hline ALT & $26 \mathrm{IU} / \mathrm{L}$ & & \\
\hline $\mathrm{LDH}$ & $203 \mathrm{IU} / \mathrm{L}$ & & \\
\hline ALP & $720 \mathrm{IU} / \mathrm{L}$ & & \\
\hline$\gamma$-GTP & $23 \mathrm{IU} / \mathrm{L}$ & & \\
\hline BUN & $21 \mathrm{mg} / \mathrm{dL}$ & & \\
\hline $\mathrm{Cr}$ & $0.94 \mathrm{mg} / \mathrm{dL}$ & & \\
\hline
\end{tabular}

vealed an uptake of ${ }^{18} \mathrm{~F}-\mathrm{FDG}$ in these lesions (Fig. 2). Because these images indicated a malignant bone disorder, a biopsy of the right radius was performed. The biopsy findings showed pathological features of diffuse large B-cell lymphoma (DLBCL) (Fig. 3). Epstein-Barr virus-encoded small RNA-1 (EBER-1) was negative. According to the WHO classification of tumors of the hematopoietic and lymphoid tissues, the diagnosis of OIIA-LPD was made (11). Because the patient did not exhibit either lymphadenopathy or visceral lesions, such as hepatomegaly and splenomegaly, she fulfilled the criteria of PBL according to the WHO classification of tumors of soft tissue and bone (12). She was admitted to the hospital after cessation of MTX for one month without regression.

A physical examination showed no superficial lymphadenopathy or hepatosplenomegaly. All laboratory data including a blood cell count, lactic acid dehydrogenase (LDH) and soluble interleukin-2 receptor (sIL-2R) were within normal ranges except for alkaline phosphatase (ALP) (Table). An iliac bone marrow biopsy showed no evidence of lymphoma infiltration. According to these findings, the clinical stage was evaluated as IVAEO+ (Ann Arbor classification) and international prognostic index (IPI) was 3 points (highintermediate risk).

We treated the patient with R-CHOP therapy (rituximab, cyclophosphamide, doxorubicin, vincristine, and prednisolone). After four cycles of this treatment, the hypointense lesions on the T1-weighted MRI image were decreased in size, but did not disappear (Fig. 4). We then added two cycles of R-EPOCH therapy (rituximab, etoposide, prednisolone, vincristine, cyclophosphamide, and doxorubicin) because the viability of the lesions was not evaluated correctly by MRI. After six cycles of chemotherapies, she achieved a complete response (CR) on PET/CT (Fig. 5), which has been maintained for more than half a year. 

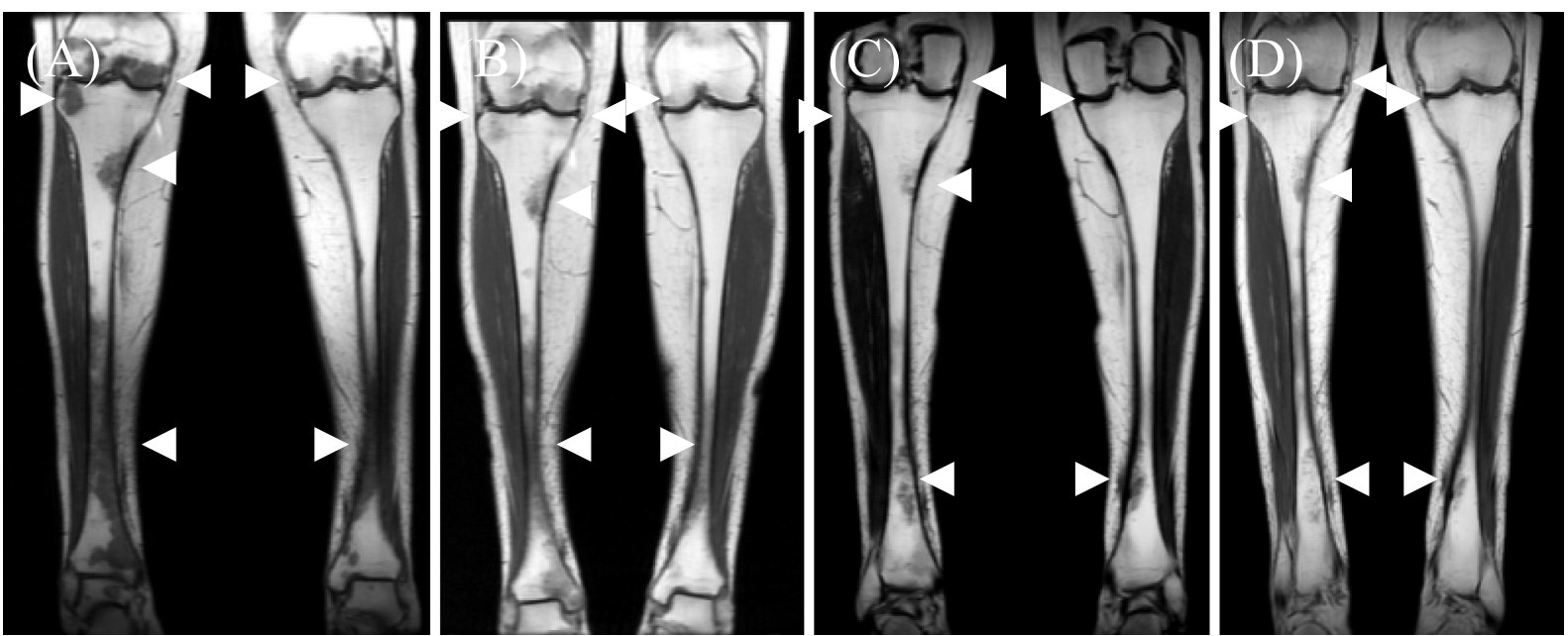

Figure 4. Hypointense lesions of the femurs and tibias on the T1-weighted MRI image were decreased after chemotherapy. (A) Before treatment, (B) after two cycles of R-CHOP therapy, (C) after four cycles of R-CHOP therapy, and (D) after four cycles of R-CHOP therapy and two cycles of REPOCH therapy. Each lesion is indicated by arrows.
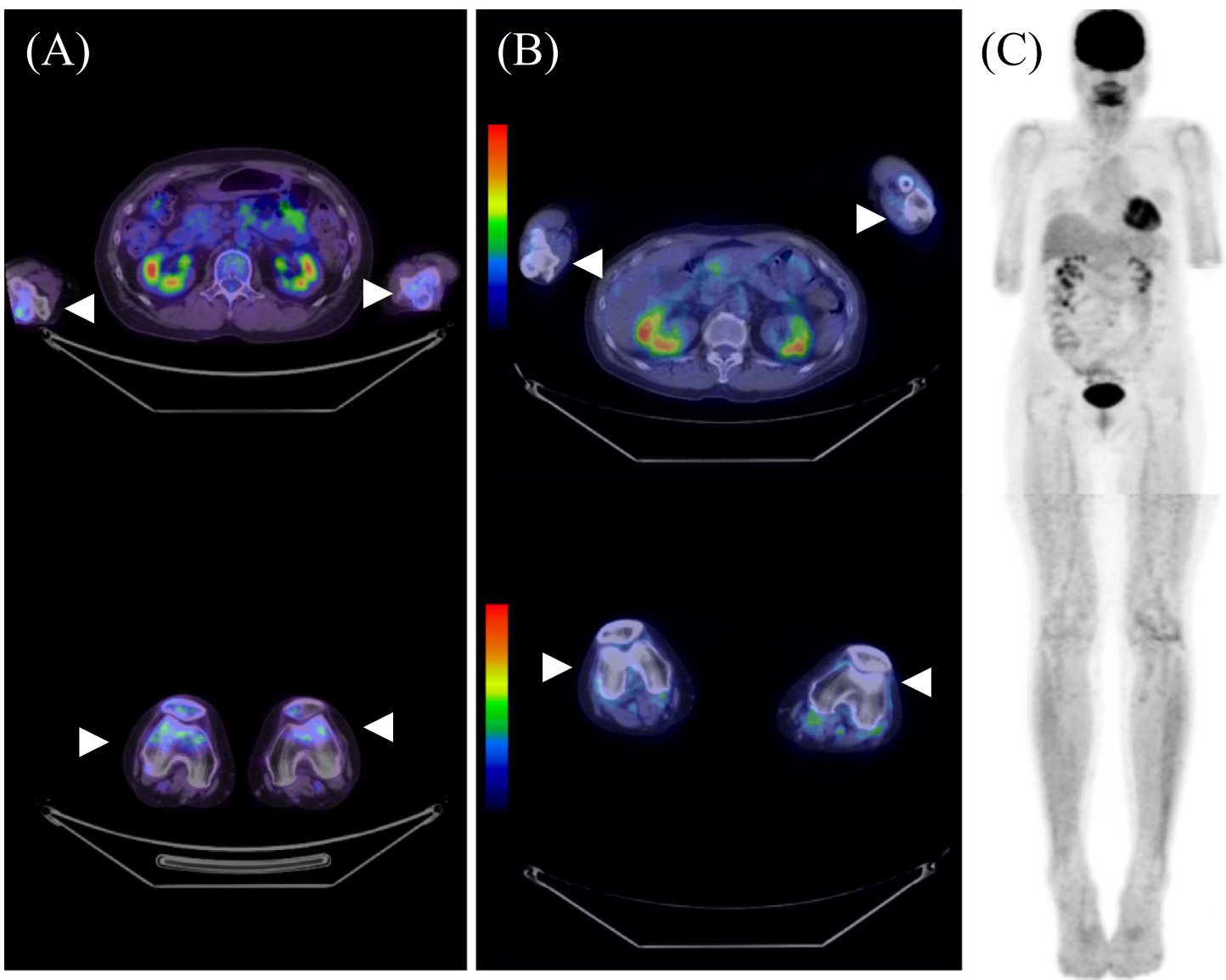

Figure 5. PET/CT images after four cycles of R-CHOP therapy and two cycles of R-EPOCH therapy did not show a significant uptake of ${ }^{18}$ F-FDG in the lesion. (A) Before treatment, (B) after six cycles of chemotherapies, (C) whole-body PET images after six cycles of chemotherapies. Each lesion is indicated by arrows.

\section{Discussion}

OIIA-LPD occasionally exhibits extranodal lesions; how- ever, the bone is rare as a primary site. PBL generally accounts for $5 \%$ of extranodal lymphomas (1-6), less than $1 \%$ of all non-Hodgkin's lymphomas $(3,6,7)$, and $3 \%$ of all primary malignant bone tumors $(2,5,8)$. PBL affects 
mainly patients in the fourth to the sixth decades of age (1$3,5-8,13-16)$ with a slight predominance in men (male:female $=1.2-1.8: 1)(1-6,13,15,16)$. In addition to the femurs and spines, Japanese studies have indicated that pelvic bones are frequently affected $(1-3,6,8,13,15-17)$. The most common histopathological subtype of PBL is DLBCL (54-92\%) $(1-8,13,14,16)$. The International Extranodal Lymphoma Study Group (IELSG) proposed the classification of PBL into four stages: stage IE, single osseous lesion; stage IIE, single osseous lesion with regional lymphadenopathy; stage IVE, multifocal bone lesions without lymph nodal or visceral disease; and stage IV, disseminated lymphoma with skeletal involvement. Stage IVE was named multifocal bone lymphoma, which constitutes $3-10 \%$ of $\operatorname{PBL}(2,18)$. The present case is compatible with this unique entity.

The etiology of PBL is unknown (6). Some factors including HIV infection, sarcoidosis, renal transplantation, and cladribine therapy have been reported in association with PBL (18). A retrospective study of LPD in patients with RA showed that the frequency of PBL was $2.6 \%$ (9). It is of note that this is the first case to report precisely it's the clinical course of PBL occurring in an RA patient treated with MTX.

The most common symptom of PBL is bone pain $(2,3$, $6-8,13,16)$. In patients with RA, it is important and occasionally difficult to distinguish pain of PBL from that of the exacerbation of RA. A physical examination, blood tests, and imaging studies, such as MRI and PET/CT, to exclude synovium inflammation of RA and to detect the bone lesion of PBL were useful in the present case. X-rays typically show focal lytic lesions or permeative destruction often with sclerotic change $(6,8,15)$. Unfortunately, a lesion of PBL may not be detected on X-rays such as in this case (8). CT is useful to evaluate soft tissue extension of $\operatorname{PBL}(6,15)$. MRI is the most sensitive imaging study in the diagnosis of PBL. The lesion typically shows iso- to hypointense on the T1-weighted image and hyperintense on the T2-weighted image $(6,8,15)$. PET/CT is useful to assess the remission status of PBL $(6,8,15,19)$. However, because all imaging study findings mentioned above are nonspecific, a bone biopsy is essential to make the diagnosis of PBL.

Due to its rarity, no standard therapy for PBL has yet been established. In the case of PBL with a single bone lesion with or without regional lymph nodes (IELSG stage IE or IIE), chemotherapy plus radiotherapy might be preferred. However, chemotherapy alone is indicated for PBL patients with multifocal bone disease (IELSG stage IVE or IV), as for this case $(1-3,6,13)$. The withdrawal of MTX reportedly caused spontaneous regression in approximately half of other iatrogenic immunodeficiency-associated lymphoproliferative disorder (OIID-LPD) cases. Spontaneous regression tended to occur in Epstein-Barr virus (EBV)-positive patients, which comprise $27.1-62.8 \%$ of OIID-LPD in Japan $(9,10)$. However, the present case was negative for EBV and the one-month withdrawal of MTX did not lead to regression. We therefore selected the R-CHOP regimen as first-line therapy.

The five-year overall survival (OS) and progression-free survival (PFS) rates of PBL were reported to be $36.7-91 \%$ and $13.4-75.7 \%$, respectively $(1-4,5-7,13,16)$. Conversely, the five-year OS and PFS rates of multifocal bone DLBCL, such as this case, were reported to be $36.7-74 \%$ and 13.4 $34 \%$, respectively $(1,2)$. Several studies have identified predictors of the prognosis of PBL. For instance, multifocality $(1,4,5)$, age $\geq 60$ years $(1,5,8)$, non-CR response (13), soft tissue extension $(3,6)$, increased $\operatorname{IPI}(3,5)$, elevated LDH (5), and B symptom positive (5) were reported to be factors for a shorter survival. The present case appeared to have a poor prognosis due to multifocality, age $\geq 60$ years and high-intermediate risk of IPI. We therefore decided to add two courses of R-EPOCH therapy after four cycles of R-CHOP therapy due to suspected insufficient responses on MRI and an estimated poor prognosis. This case achieved a CR on PET/CT after six cycles of chemotherapies and the $\mathrm{CR}$ has been maintained for more than half a year.

This case elucidates the importance of considering PBL in patients with RA complaining of bone pain. MRI and FDGPET/CT confer clues for the diagnosis and biopsy site. A histologic examination of the biopsy specimen is mandatory for the diagnosis of lymphoma. This report is the first documentation of the clinical course of an OIIA-LPD case presenting as multifocal PBL.

\section{The authors state that they have no Conflict of Interest (COI).}

\section{References}

1. Wu H, Bui MM, Leston DG, et al. Clinical characteristics and prognostic factors of bone lymphomas: focus on the clinical significance of multifocal bone involvement by primary bone large B-cell lymphomas. BMC Cancer 14: 900, 2014.

2. Messina C, Ferreri AJ, Govi S, et al. Clinical features, management and prognosis of multifocal primary bone lymphoma: a retrospective study of the international extranodal lymphoma study group (the IELSG 14 study). Br J Haematol 164: 834-840, 2014.

3. Wu H, Zhang L, Shao H, et al. Prognostic significance of soft tissue extension, international prognostic index, and multifocality in primary bone lymphoma: a single institutional experience. $\mathrm{Br} \mathrm{J}$ Haematol 166: 60-68, 2014.

4. Jawad MU, Schneiderbauer MM, Min ES, Cheung MC, Koniaris LG, Scully SP. Primary lymphoma of bone in adult patients. Cancer 116: 871-879, 2010.

5. Ramadan KM, Shenkier T, Sehn LH, Gascoyne RD, Connors JM. A clinicopathological retrospective study of 131 patients with primary bone lymphoma: a population-based study of successively treated cohorts from the British Columbia Cancer Agency. Ann Oncol 18: 129-135, 2007.

6. Kitsoulis P, Vlychou M, Papoudou-Bai A, et al. Primary lymphomas of bone. Anticancer Res 26: 325-337, 2006.

7. Horsman JM, Thomas J, Hough R, Hancock BW. Primary bone lymphoma: a retrospective analysis. Int J Oncol 28: 1571-1575, 2006.

8. Zhou HY, Gao F, Bu B, et al. Primary bone lymphoma: a case report and review of the literature. Oncol Lett 8: 1551-1556, 2014.

9. Hoshida Y, Xu JX, Fujita S, et al. Lymphoproliferative disorders in rheumatoid arthritis: clinicopathological analysis of 76 cases in re- 
lation to methotrexate medication. J Rheumatol 34: 322-331, 2007.

10. Ichikawa A, Arakawa F, Kiyasu J, et al. Methotrexate/iatrogenic lymphoproliferative disorders in rheumatoid arthritis: histology, Epstein-Barr virus, and clonality are important predictors of disease progression and regression. Eur J Haematol 91: 20-28, 2013.

11. Gaulard P, Swerdlow SH, Harris NL, et al. WHO Classification of Tumours of Haematopoietic and Lymphoid Tissues. International Agency for Research on Cancer, Lyon, France, 2008: 350-351.

12. Fletcher CD, Bridge JA, Hogendoorn P, et al. WHO Classification of Tumours of Soft Tissue and Bone. International Agency for Research on Cancer, Lyon, France, 2013: 316-318.

13. Liu YC, Gau JP, Yu YB, et al. Prognostic factors and treatment efficacy in patients with primary diffuse large B-cell lymphoma of the bone: single institute experience over 11 years. Intern Med 53: 95-101, 2014

14. Bhagat P, Sachdeva MU, Sharma P, et al. Primary bone marrow lymphoma is a rare neoplasm with poor outcome: case series from single tertiary care centre and review of literature. Hematol Oncol
34: 42-48, 2016.

15. Misgeld E, Wehmeier A, Krömeke O, Gattermann N. Primary non-Hodgkin's lymphoma of bone: three cases and a short review of the literature. Ann Hematol 82: 440-443, 2003.

16. Demircay E, Hornicek FJ Jr, Mankin HJ, Degroot H 3rd. Malignant lymphoma of bone: a review of 119 patients. Clin Orthop Relat Res 471: 2684-2690, 2013.

17. Maruyama D, Watanabe T, Beppu Y, et al. Primary bone lymphoma: a new and detailed characterization of 28 patients in a single-institution study. Jpn J Clin Oncol 37: 216-223, 2007.

18. Messina C, Christie D, Zucca E, Gospodarowicz M, Ferreri AJ. Primary and secondary bone lymphomas. Cancer Treat Rev 41: 235-246, 2015.

19. Park YH, Kim S, Choi SJ, et al. Clinical impact of whole-body FDG-PET for evaluation of response and therapeutic decisionmaking of primary lymphoma of bone. Ann Oncol 16: 1401-1402, 2005.

(C) 2016 The Japanese Society of Internal Medicine

http://www.naika.or.jp/imonline/index.html 